\title{
Crack Classification and Interpolation of Old Digital Paintings
}

\author{
Shruti Garg ${ }^{1, *}$, G. Sahoo ${ }^{2}$ \\ ${ }^{1}$ Department of CSE, BIT, Mesra, Ranchi, Jharkhand, India \\ ${ }^{2}$ Department IT, BIT, Mesra, Ranchi, Jharkhand, India \\ *Corresponding author: gshruti_garg@yahoo.com
}

Received May 06, 2013; Revised June 25, 2013; Accepted June 27, 2013

\begin{abstract}
Paintings which was handled roughly or made from low quality paint or base usually suffers from crack in a long run, which causes them to lose some of the information. This paper discuss about automatic approach for classification and interpolation of cracks. For classification supervised and unsupervised methods were implemented and for interpolation different order statistics filter were applied. Experimental result shows that unsupervised classification works better than supervised classification. And variable size window filter works best for interpolation of cracks.
\end{abstract}

Keywords: crack detection, top-hat transform, median filter, trimmed filter, back propagation algorithm, fuzzy cmeans algorithm

Cite This Article: Garg, Shruti, and G. Sahoo, "Crack Classification and Interpolation of Old Digital Paintings." Journal of Computer Sciences and Applications 1, no. 5 (2013): 85-90. doi: 10.12691/jcsa-1-5-2.

\section{Introduction}

Image restoration, which is also known as image interpolation problem, has wide applications in image processing such as film and photo restoration, text removal, special effects in movies disocclusion, digital zoom-in, and restoration of old art works etc. Present paper focuses on finding the appropriate positions of cracks and removal of them. A system that is capable of tracking and interpolating cracks is presented in [1] but it requires the user to manually start with the initial point of the crack pattern to fill them. Another method for the detection of cracks using multi-oriented Gabor filters is presented in [2]. Crack detection and removal is however similar with the methods proposed for the detection and removal of scratches and other artifacts from motion picture films $[3,4,5]$ but these methods uses the information obtained over several adjacent frames for both detection and filling and thus are not applicable for the cracks in paintings. Another similar approach was also specified by Solanki and Mahajan [22] but it is semiautomatic approach, again user intervention is required at stage of classification.

In this work emphasis is given to the classification of cracks because the interpolation quality will be solely depends on the classification. Classification is done by two different approaches supervised and unsupervised and both the methods are fully automatic. Before applying classification step crack detection was applied first to separate out low eliminated areas of digital paintings, discussed in section 2. Section 3 describes the methods for classification and in section 4 crack interpolation methods were described which decides quality of classification. Section 4 discussed about results and discussions and the in last section is conclusion.

\section{Detection of Cracks}

Cracks are generally low eliminated and elongated in nature. Therefore, a crack detector can be applied on the luminance component of an image and should be able to identify such minima was presented in [7]. The detection of the cracks can be obtained with the implementation of a very useful morphological filter, called top-hat transformation. The top-hat transform which is described in [8] is a grayscale morphological filter defined as follows:

$$
y(x)=f(x)-f_{n B}(x)
$$

where $f_{n B}(x)$ is the opening of the function with the structuring set $\mathrm{nB}$, defined as:

$$
n B=B \oplus B \oplus B \oplus B \ldots . . B \text { (n rimes) }
$$

The opening $f_{n B}(x)$ of a function is a low-pass nonlinear filter that erases all peaks (local maxima) in which the structuring element $\mathrm{nB}$ cannot fit. Thus, the image $f-f_{n B}$ contains only those peaks and no background at all. The cracks have usually very small luminance. Thus, for extracting the cracks, we must negate the luminance image and then apply the top-hat transformation. Alternatively, crack can also be detected by performing closing on the original image $f(x)$ with the 
structuring set $\mathrm{nB}$ and then subtracting $f(x)$ from the result of closing $f^{n B}(x)$.

$$
y(x)=f^{n B}(x)-f(x)
$$

For our results the type of structuring element is square type whereas the size of structuring element that is used is $3 \times 3$ and the number of times dilation operation is applied on the image is 2 as the thickness of cracks ranges from 2 to 6 pixels. The top-hat transform generates a grayscale output image $t(k, l)$ where pixels with a large grey value are potential crack or crack-like elements. Therefore, a thresholding operation on $t(k, l)$ is required to separate cracks from the rest of the image. The threshold $\mathrm{T}$ can be chosen by inspecting the top-hat output histogram, so that only a small percentage of the pixels $t(k, l)$ is above it. The thresholding is global, because $\mathrm{T}$ is chosen based on global information is given in [7]. In this case the threshold value $\mathrm{T}$ is taken as 23 which is providing good results. The result of the thresholding is a binary image $b$ $(k, l)$ marking the possible crack locations which generates the crack map.

\section{Crack Classification}

Because top hat transform works as low pass filter, it passes all low frequencies that may be a thin brush stroke or crack. So the brush strokes may be misclassified as cracks in crack detection step. Thus, in order to avoid any undesirable alterations to the original image, it is very important to separate these brush strokes from the actual cracks, before the implementation of the crack filling procedure. Two methods to achieve this goal are described as follows:

\subsection{Supervised Crack Classification using Back propagation Algorithm}

It has been observed by analyzing some paintings that the gray values of cracks usually ranges from 0 to 122.4 while the gray values of the brush strokes may vary from 0 to 255 as brush strokes of any painting can be any value depending on the color of paint. Thus, a great portion of the dark brush strokes, falsely detected by the top-hat transform can be separated from the cracks by using this information. This separation can be achieved by classification using a Back propagation neural network.

Back propagation [9] is a feed forward neural network which usually consists of three or more layers: input, output and hidden layer. And it works on training of previous patterns. Weights were updated during training phase. And next phase is the testing phase, where no learning takes place. Each test vector is fed into the input layer and results will be obtained on the basis of stored weights at training phase.

In this paper, back propagation neural network generates two outputs. The first output represents the class of cracks while the second one the class of brush strokes. Input vectors fed to the network are two-dimensional and consisted of the gray values of pixels identified as cracks by the top-hat transform.

\subsection{Unsupervised Crack Classification by Fuzzy C-means Algorithm}

In unsupervised classification training data sets is not required, the data is divided in clusters. There will be always two clusters in this case, one is for cracks and another is for brush strokes. The input for fuzzy c- mean clustering was the output of top hat transform

\section{Crack Filling}

After identifying cracks and separating misclassified brush strokes, the final task is to restore the image using local image information (i.e., information from neighboring pixels) to fill (interpolate) the cracks. This step will also assure about the quality of classification. Different approaches for interpolating information were discussed in [15-20]. The technique here used for interpolation of cracks is order statistics filter. Cracks are missing pixel values in paintings, in order to generate them order statistics filters were applied, because they gives a similar values as their neighbors are having.

\subsection{Trimmed Mean Filter}

A trimmed mean filter excludes the samples $x_{i+r, j+s}$ in the filter window, which is considerably smaller from the local median and averages the remaining pixels.

$$
y_{i j}=\left(\sum \sum_{A} \propto_{r s} x_{i+r, j+s}\right) /\left(\sum \sum_{A} \propto_{r s}\right)
$$

where,

$$
\propto_{r s}=\left\{\begin{array}{rr}
0, & \text { if med }\left\{x_{i j}\right\}-x_{i+r, j+s} \geq q \\
1, & \text { otherwise }
\end{array}\right.
$$

where, $\mathrm{q}$ is a positive parameter.

\subsection{Modified Trimmed Mean Filter}

Modified trimmed mean filter performs mean filtering only on those pixels that are not part of the crack, i.e., it utilizes information from the binary output image $\mathrm{b}(k, l)$ of the top-hat transform. In this case, the filter coefficients as follows:

$$
\propto_{r s}=\left\{\begin{array}{lr}
0, & \text { if } b(k, l)=1 \\
1, & \text { otherwise }
\end{array}\right.
$$

\subsection{Alternative Mean Median Filter}

The previous two methods gives smoothing effects. And fixed numbers of neighbors were considered in them. But cracks are not always of same width. At some places there will be thin crack and there may be a wider crack at another place. In order to fill them all with less smoothing effect window size will vary in this method. Firstly $13 \times$ 13 mean filter is applied on the crack pixels followed by 3 $\times 3$ median filter. Recursively size of mean filter will decrease, e.g. $9 \times 9$ mean filter and size of median filter will increase, e.g. $5 \times 5$ median filter.

\subsection{Variable Median Filter}


The problem with the standard median filter method lies on its fixed window size and that there could always be a possibility that crack pixel count in the local region may exceed the non crack pixel count. This may however, result in replacing a crack pixel by another crack pixel, thus, failing in our aim. Therefore median filter with varying window size surrounding the crack pixel were used. The variations depend on the nature of pixels surrounding the crack pixels in the local region of window. If window is have more than $50 \%$ of crack pixels then window size will be increased to $5 \times 5$ and soon.

\section{Results}

An integrated strategy for crack classification and filling had been presented for digitized paintings. Cracks are detected by using top hat transform and thresholding operation on cracked painting. The parameters chosen for this technique are: Structuring element as square, size as $3 \times 3$ and number of dilations (n) as 2. After top hat transform the result is thresholded using the threshold value 23. This will generate the following results:

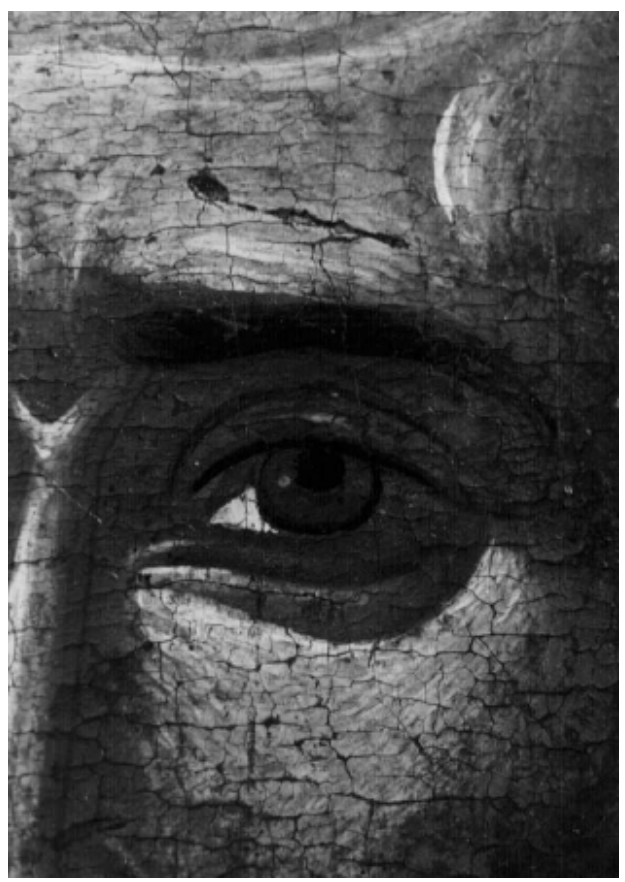

Figure 1. Original Image

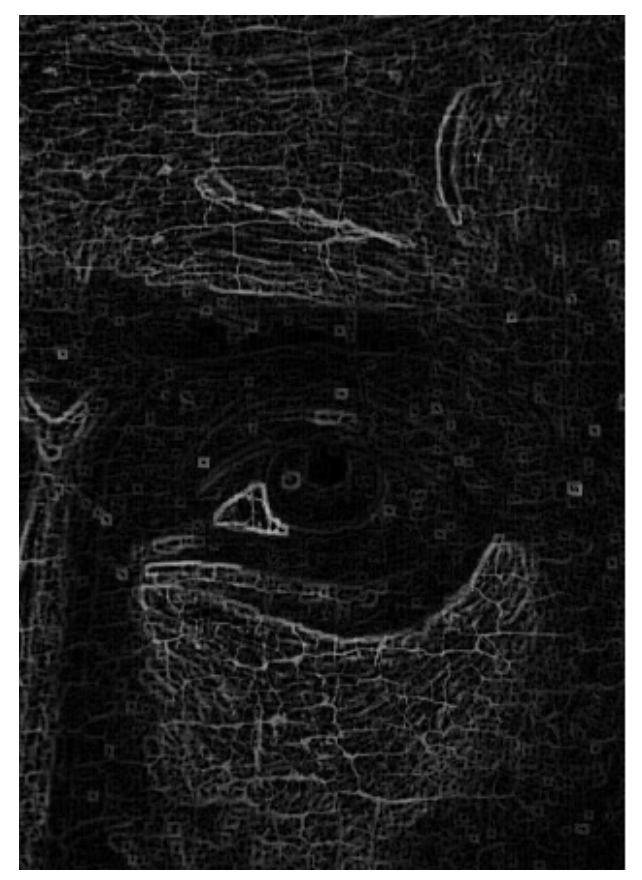

Figure 2. Result after applying top hat transform

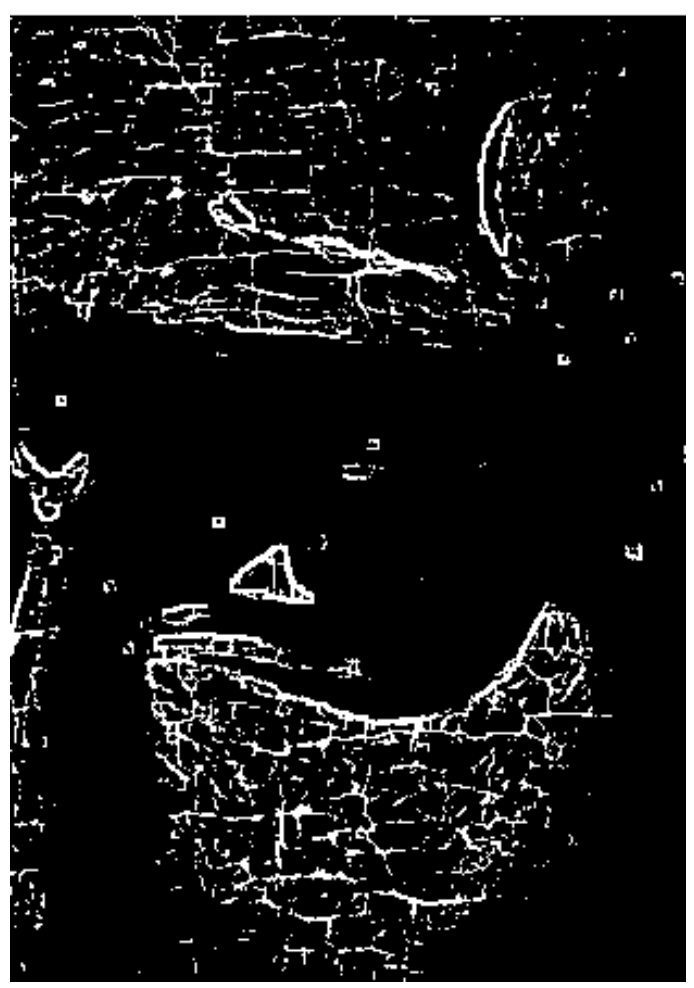

Figure 3. Crack map after applying thresholding

The thin dark brush strokes, which are misidentified as cracks, are separated either by back propagation neural network or by fuzzy c mean clustering approach. The methods and their results that are used for the separation of misidentified cracks are as follows:

\subsection{Crack Classification}

\subsubsection{Supervised Crack Classification using Back Propagation Algorithm}

The back propagation neural network is implemented on the gray values of the cracked digitized painting using the information from the binary output image $b(k, l)$ of the top-hat transform. 


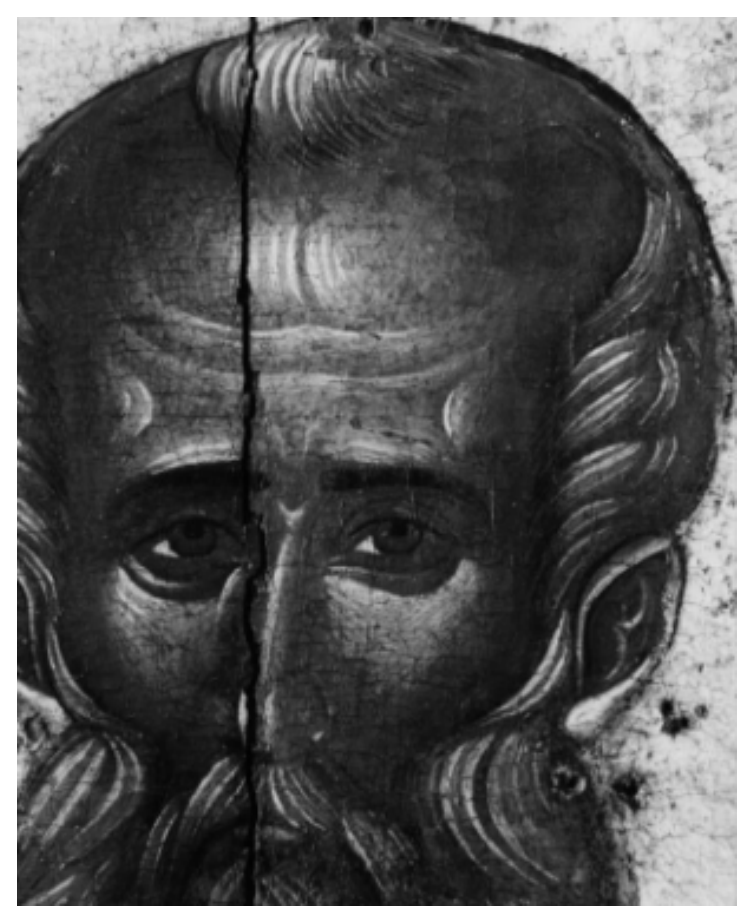

Figure 4. Original Image

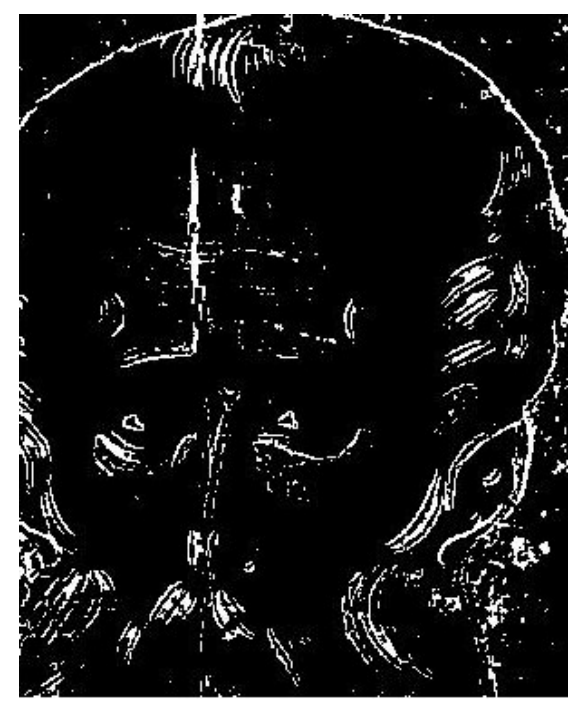

Figure 5. Crack Map before classification

5.1.2. Unsupervised Crack Classification by Fuzzy CMeans Algorithm

The fuzzy partition is also implemented on the gray values of the cracked digitized painting using the information from the binary output image $b(k, l)$ of the tophat transform. This will generate the following results:

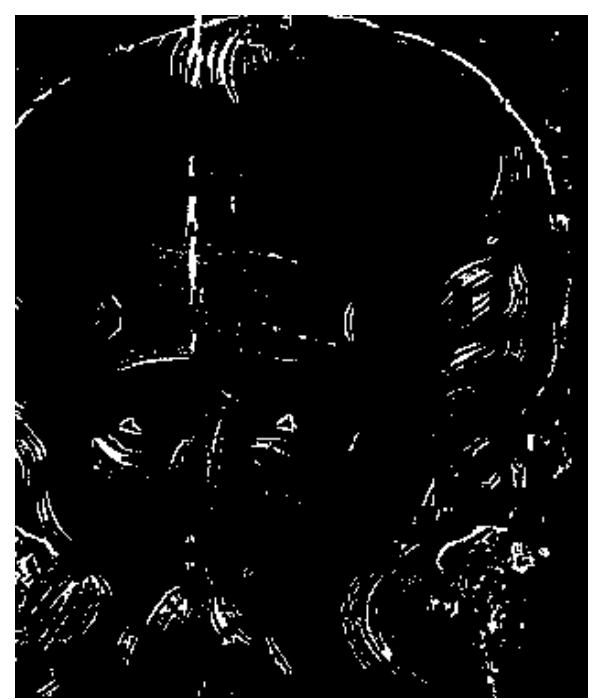

Figure 6. Crack Map after supervised classification

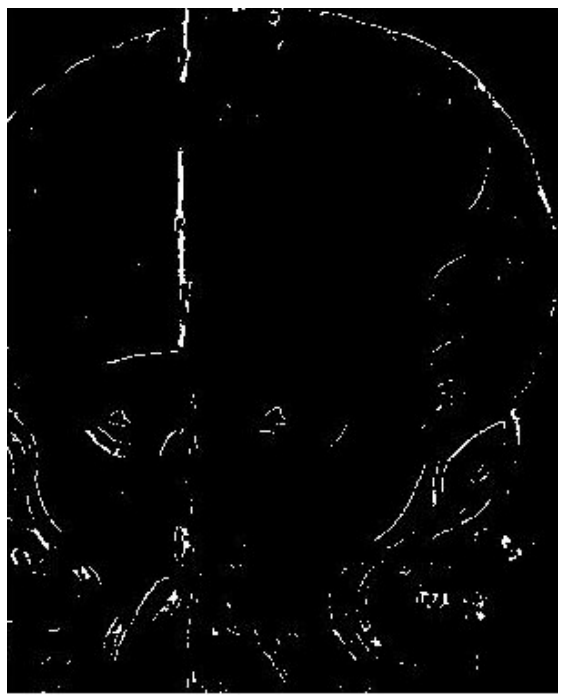

Figure 7. Cracks Map after unsupervised

\subsection{Crack Interpolation}

Crack interpolation is performed appropriately by modified order statistics filters. The methodology has been applied for the virtual restoration of images and was found very effective. The methods and their results that are used for interpolation are as follows:

\subsubsection{Modified Trimmed Mean Filter}




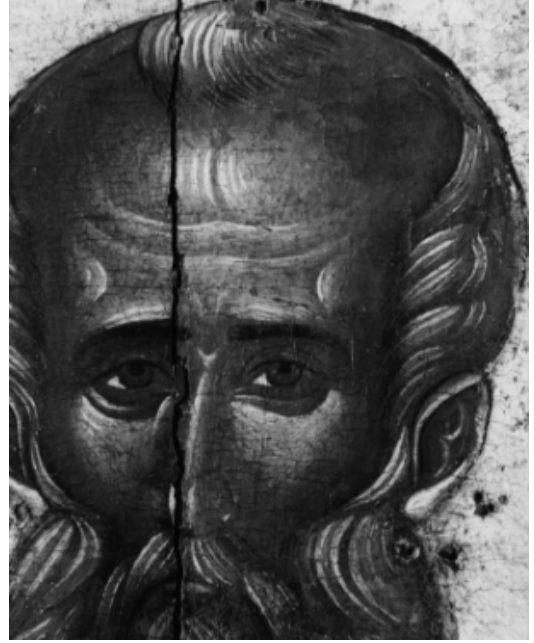

Figure 8. Original Image

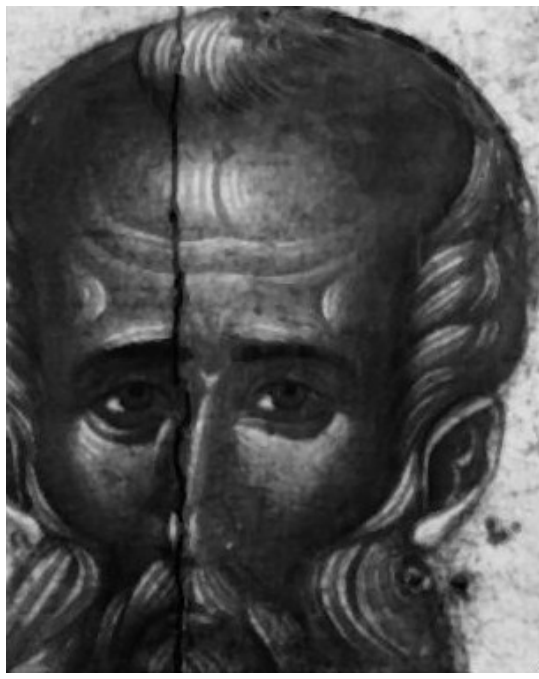

Figure 9. Result after applying Modified Trimmed Mean Filter

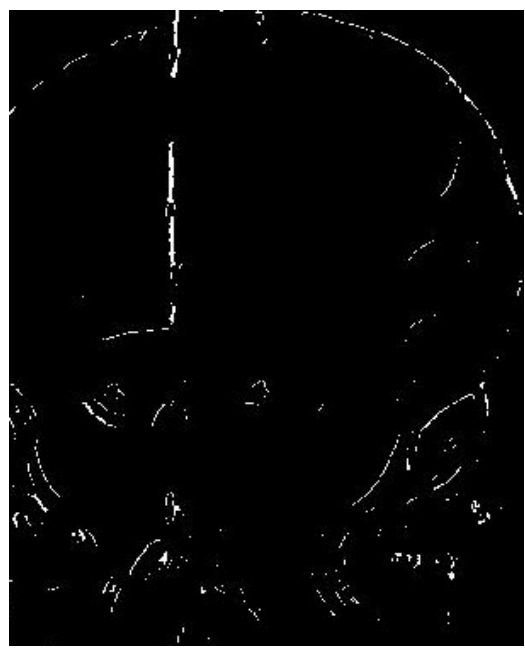

Figure 10. Crack Map before interpolation

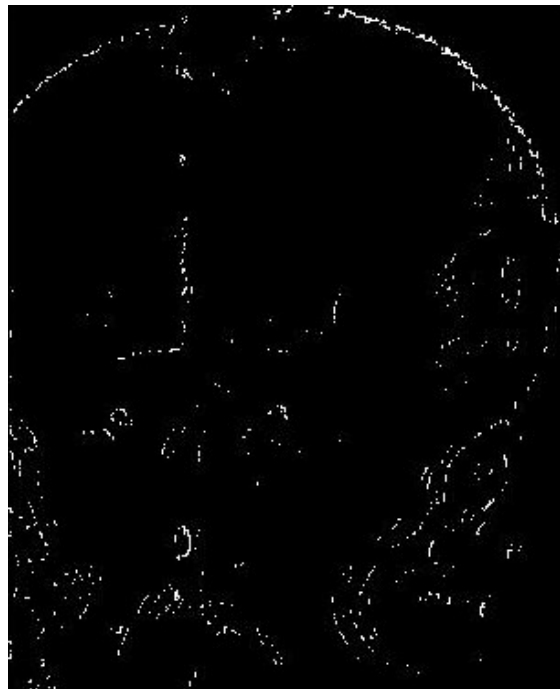

Figure 11. Crack map after interpolation

\subsubsection{Trimmed Mean Filter}

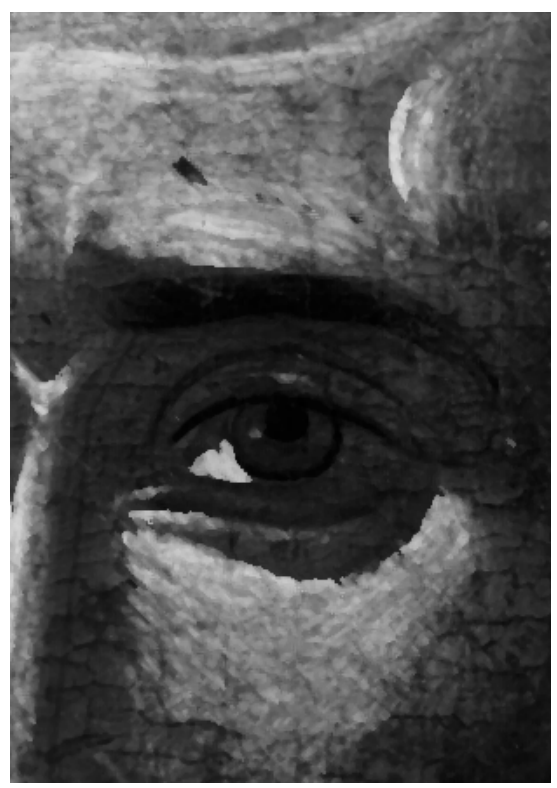

Figure 12. Result after applying Trimmed Mean Filter

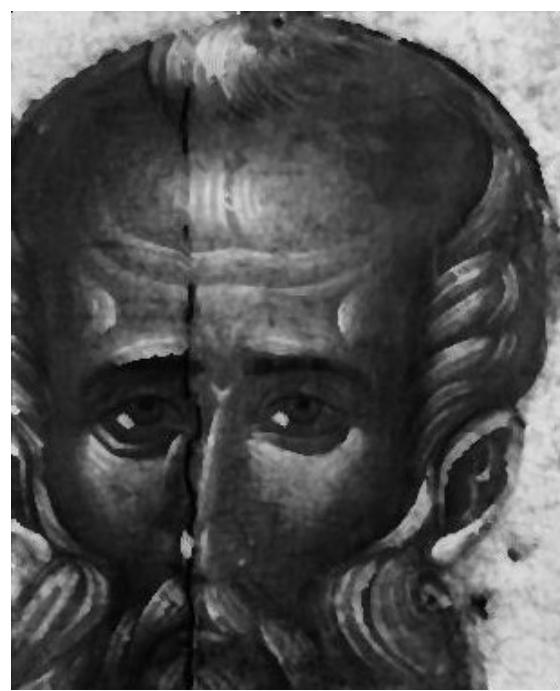

Figure 13. Result after applying Trimmed mean Filter

\subsubsection{Alternative Mean Median Filter}




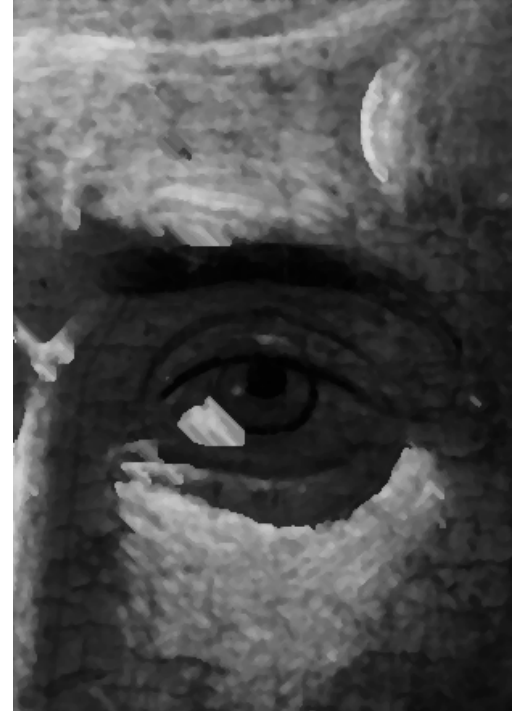

Figure 14. Result after applying Alternative Mean Median Filter

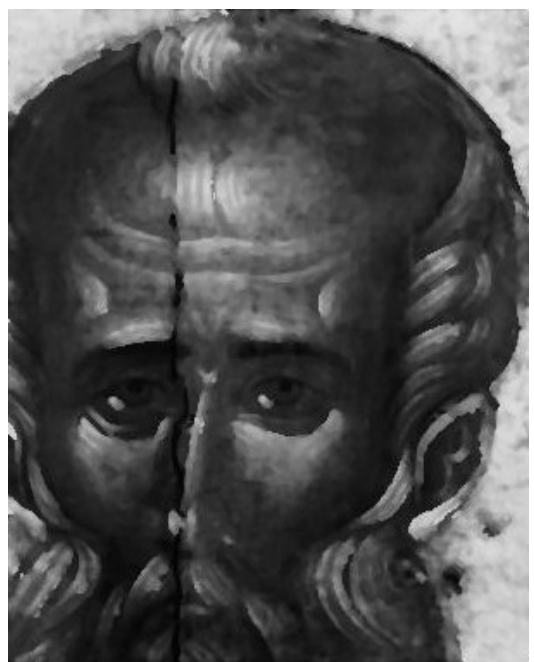

Figure 15. Result after applying Alternative Mean Median Filter

Alternative mean median filter or trimmed mean filter or trimmed median filter does not depend upon the number of crack pixels in the filter window. And thus it may replace the crack pixel with another crack pixel. Therefore, a variable size median filter can be used taking account the number crack pixels in the window.

\subsubsection{Variable Size Median Filter}

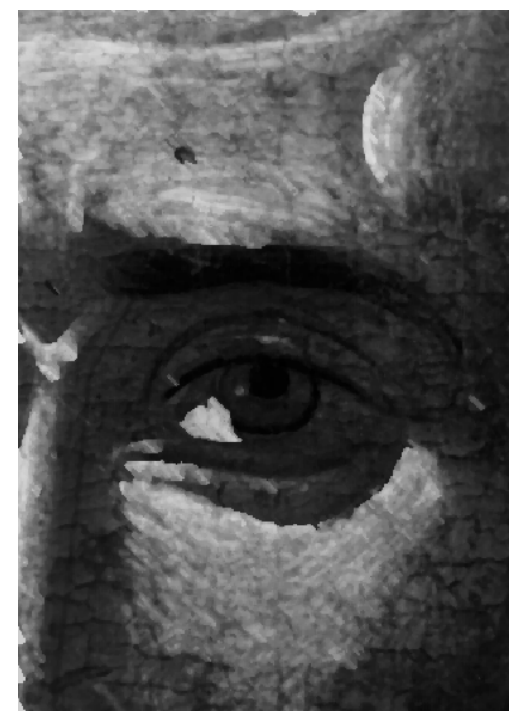

Figure 16. Result after applying Variable size median filter

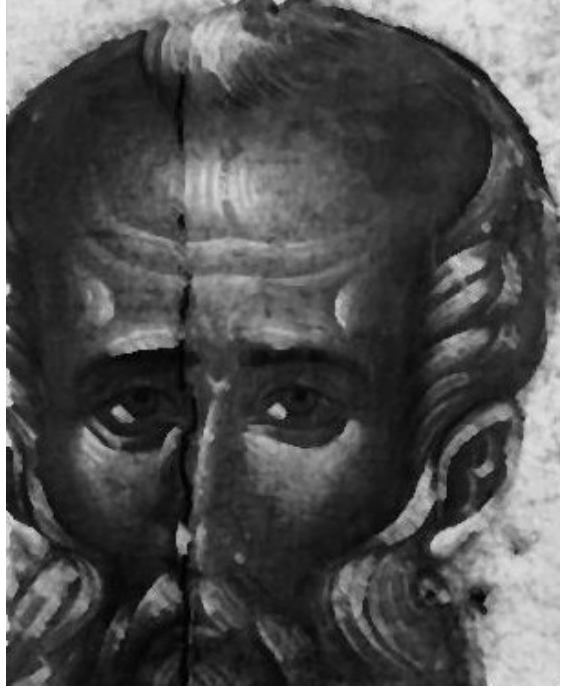

Figure 17. Result after applying Variable size Median Filter

\section{Conclusion}

The work presented is automatic methodology for detection of cracks in old digitalized painting. The results presented in this work regard the craquelure of old paintings, however, the same algorithms can be used for a much wider set of application. Two different algorithms were applied for classification in present work supervised and unsupervised method. Experimental results show that, fuzzy c-Means algorithm works well in most of the cases and gives the better output then back propagation. Also wider cracks were not filled by mean or modified mean approach. The variable size median and alternative mean median gives better results in case of wider cracks.

\section{References}

[1] Barni, M., Bartolini, F., Cappellini, V., "Image processing for virtual restoration of artworks," IEEE Multimedia, 7(2). 34-37. April-June 2000.

[2] Abas, F., Martinez, K., "Craquelure Analysis for Content-based Retrieval," in Proceedings of 14th International Conference on Digital Signal Processing , IEEE, 1, 111-114, 2002.

[3] Joyeux, L., Buisson, O., Besserer, B., Boukir, S., "Detection and removal of line scratches in motion picture films," in Proceedings of International Conference on Computer Vision and Pattern Recognition, IEEE, 548-553, 1999.

[4] Kokaram, A., Morris, R., Fitzgerald, W., Rayner, P., "Detection of missing data in image sequences," IEEE Transactions on Image Processing, 4(11). 1496-1508. November 1995.

[5] Kokaram, A., Morris, R., Fitzgerald, W., Rayner, P., "Interpolation of missing data in image sequences," IEEE Transactions on Image Processing, 4(11). 1509-1519. November 1995.

[6] Giakoumis, I., Nikolaidis, N., Pitas, I., "Digital Image Processing Techniques for the Detection and Removal of Cracks in Digitized Paintings," IEEE Transactions on Image Processing, 15(1). 178188. Jan. 2006.

[7] Giakoumis, I., Pitas, I., "Digital Restoration of Painting Cracks" in International Symposium on Circuits and Systems (ISCAS' 98), IEEE, California USA, June 1998.

[8] Meyer, F., "Iterative image transforms for an automatic screening of cervical smears," Journal Histoch. Cytochem, 27, 128-135, 1979.

[9] Rajashekharan, S.: Neural Network, Fuzzy Logic and Genetic Algorithms-Synthesis and Application, Prentice Hall, Wiley, 2005, 34-86. 
[10] Karray, F.: Soft Computing and Intelligent System Design-Theory, Tools and Applications, Addison Wesley, New York, 2005, 249293.

[11] Dubois, D., Prade, H.: Fundamentals of fuzzy sets, Kluwer Academic Publishers, The Netherlands, 2000.

[12] Fuller, R., "On product-sum of triangular fuzzy numbers,” Fuzzy Sets and Systems, 41(1), 83-87, 1991.

[13] Zadeh, L. A., "Fuzzy sets," International Journal of Information and Control, 8, 338-353, 1965.

[14] Bertalmio, M., Sapiro, G., Caselles, V., Ballester C., "Image inpainting," in Proceedings of SIGGRAPH, ACM, 417-424, 2000.

[15] Ballester, M., Bertalmio, V., Caselles, G., Sapiro, G., Verdera J., "Filling-in by joint interpolation of vector fields and gray levels," IEEE Transaction of Image Processing, 10(8), 1200-1211, Aug 2001.

[16] Masnou, S., Morel, J. M.," Level lines based disocclusion,” Proceedings of International Conference of Image Processing, IEEE, 3, 259-263, 1998.
[17] Chan, T., Shen, J., "Non-texture inpaintings by curvature-driven diffusions," Journal of Visual Communication and Image Representation, 12,(4), 436-449, 2001.

[18] Esedoglu, S., Shen, J., "Digital inpainting based on the MumfordShah-Euler image model," European Journal of Applied Mathematics, 13, 353-370, 2002.

[19] Efros, A., Leung, T., "Texture synthesis by nonparametric sampling," Proceedings of International Conference of Computer Vision, IEEE 1033-1038, 1999.

[20] Jha, R., Garg, S., Sahoo, G., "Crack Filling in Old Digital Paintings," International Journal of Graphics and Image Processing, 1(2), 210-214, 2011.

[21] Solanki, S. V., Mahajan, A. R., "Cracks Inspection and Interpolation in Digitized Artistic Picture using Image Processing Approach," International Journal of Recent Trends in Engineering, 1(2), 97-99, May 2009. 